\title{
A Framework for Software Development Technical Reviews
}

\author{
Lesley Pek Wee Kim ${ }^{a}$, Chris Sauer ${ }^{b}$, and Ross Jeffery ${ }^{\mathrm{c}}$ \\ ${ }^{\text {a }}$ School of Computer Science and Engineering \\ ${ }^{b}$ Fujitsu Centre, Australian Graduate School of Management \\ ${ }^{\mathrm{c}}$ School of Information Systems
}

The University of New South Wales, Sydney 2052, Australia.

\begin{abstract}
Reviews are seen as an important aid to achieving high quality software. But, there is considerable variance in the literature. Three seminal review types - Fagan's inspection, Freedman and Weinburg's technical review, and Yourdon's structured walkthrough are briefly described. A framework is presented to identify core features and variants. The core components represent an implicit normative theory of reviews; the variants indicate possible variations to the theory. There are indications emerging that this theory may be flawed. This framework provides a foundation from which to empirically test the core components of the theory and its variants.
\end{abstract}

Keyword Codes: D.2, D.2.0, D.2.9

Keywords: software engineering, quality, inspection, structured walkthrough, technical review.

\section{INTRODUCTION}

Software engineering is said to be 'a strategy for producing quality software' [5]. It is widely agreed that the traditional approach of defect testing just before product delivery is far too late [1], especially if we consider the popular $6 \Sigma$ characterisation of quality which translates to approximately 3 to 4 defects per million lines of code [8]. The software review technique is seen as a method of removing errors throughout the software life cycle. The literature offers competing prescriptions. These prescriptions appear to be similar in a number of basic features, but detailed examination reveals many differences. It is possible that these variations complicate the application of reviews within organisations. Moreover, researchers in the field have no common ground on which to base their research because it is not clear what is essential and what is inessential in the differing prescriptions. This paper attempts to build a framework to remedy this by analysing three seminal review types. Section 2 summarises each of the review types. Section 3 describes the SDTR (Software Development Technical Reviews) framework, via the common and different characteristics of the review types. Finally, we conclude the paper by outlining the contributions of this framework, and giving suggestions for future research in the area.

\section{THE THREE REVIEW TYPES}

Three major review types stand out in the literature: Fagan's inspection (FI), Freedman and Weinburg's technical review (FWTR), and Yourdon's structured walkthrough (YSW). They have been selected for their completeness and high volume of citation. This section summarises them. 


\subsection{Fagan's Inspection}

Fagan's inspection (1972) can be tailored to control any software quality attribute [9]. Major claimed quantitative benefits are error detection, cost effectiveness, productivity, software quality, predictability, and information on development operations. Nonquantitative benefits include better service to users, and a professional development environment.

Among recommendations for the structure of FI, an important part is the presence of a good moderator [1,2]. Each of the other reviewers plays a role: author, reader, or tester. Management's presence in meetings need not adversely affect review outcomes, but reviews should not be used for staff appraisal [2]. Recommended FI team size is 4, but can vary with the review object. Prescriptions for reviewer training are also given [1]. The inspection process can be divided into six operations [1]: planning (moderator plans the meeting), overview (meeting for product education, document distribution, role assignment), preparation (reviewer preparation), inspection (meeting for defect finding, reader paraphrases the object), rework (error correction), and follow-up (moderator ensures proper rework, determines need for reinspection).

The FI process thus consists of pre-inspection activities, the FI meeting, and post-inspection activities. It contains at least one meeting, the aim of which is to find defects only [1]. Error classes and checklists assist reviewing. The FI process must have a well-defined software development process, and a proper description and execution of FI at each stage. FI serves to detect errors, provides error control, and provides feedback to the producer. It was first devised for design and code, but can be tailored for any development product. Specification, design changes, unit test, test case, and documentation [2] can also be inspected. The meeting duration should be a maximum of 2 hours [2]. The physical outputs are reports on defects, and meeting details. They are detailed and of fixed formats, and enable management to perform statistical analysis of the reviews. A database exists to record defect classes [1]. Other matters discussed include starting inspections, defect definitions, prerequisites for using FI, and inspection scheduling.

\subsection{Freedman and Weinburg's Technical Review}

The claimed quantitative benefits are the detection and correction of errors, reduction of errors reaching testing stage, and reduction in maintenance costs. Nonquantitative benefits claimed include providing management with reliable technical information for project control, education for reviewers, communication between reviewers, and favourable effects and appreciation on staff competence.

The structure of FWTR includes role assignments: review leader, recorder, and reviewers. Two types of roles are not differentiated in the literature: project and meeting roles. A project role relates to project responsibilities (eg designer). A meeting role relates to meeting responsibilities (eg moderator). Hence FWTR has meeting roles only and has no reader or presenter. Managers usually do not participate in meetings [3]. It is not specified whether FWTR can be used for staff evaluation. Team size should be kept small (minimum 2). The same team should be used throughout a project [3]. No formal reviewer training is mentioned (except for review leader); reviewers are best trained by experience [3]. The FWTR process consists of pre-FWTR activities (planning, preparation), meeting activities, and post-FWTR activities (report production and distribution). Review aids exist for the review leader and recorder [3,5]. Anything tangible used in software development can be reviewed [3]. A review object can be broken down by form (seeking different perspectives on the same material) and by function (breaking it into smaller pieces) $[3,5]$. The meeting should last 2 hours maximum[3]. A review summary report, issues list (addressed by producer), and the related issues report are produced. The latter two are kept in the history file. Recommendations are given on the use of history file [3,5]. Other matters discussed include error explanations, scheduling [3,5], starting reviews, and physical recorder placement. 


\subsection{Yourdon's Structured Walkthrough}

Yourdon's structured walkthrough is viewed as a natural complement to the software structured development techniques [8]. The purpose is to get a group of people from roughly the same level to scrutinise a piece of work from different perspectives. The review is 'structured' so that it may be conducted in an organised way. The stated YSW quantitative benefits are error detection and establishment of standards [8]. Nonquantitative benefits claimed are providing training to staff and 'insurance'[8] for incomplete work (more chance to salvage work when someone leaves the project).

YSW requires project and meeting roles: presenter, coordinator, secretary/scribe, maintenance oracle, standards bearer, user representative, and outsider [8]. Team size should be 5 or 6 [8]. No reviewer training is mentioned. Yourdon discusses the psychological games played in walkthroughs and how they affect the outcome $[5,8]$. Yourdon also provides guidelines for forming programming teams $[5,8]$. Management roles and staff performance evaluation are also discussed [8]. The overall structure of the YSW process consists of pre-YSW activities (planning, preparation), meeting activities, and post-YSW activities (report production, error correction). Analysis, design and code guidelines are tailored for structured methods only. Standards are used to aid reviews. YSW objects are either product-related, or operational prototypes [8]. The meeting should be 30 minutes minimum, and 2 hours maximum [8]. Outputs consist of a public meeting summary, and an action list (addressed by producer) [8]. Other matters discussed include the varying degrees of formality of YSW $[5,8]$, cases when YSW are not worthwhile $[5,8]$, use of structured methodologies, prescriptions for reviewers' responsibilities, and defects.

\section{THE SDTR FRAMEWORK}

The existing literature on reviews consists of many publications originating mostly from the three review types we have described $[1-3,6,8,10-16]$. The major purpose of this paper then is to expose the principles underlying these review types. We accomplish this by scrutinising the literature in search of their core similarities and differences. The resulting SDTR framework consists of a dichotomy of review features: key features (common to the three review types) and significant variations (differing in at least one of the three review types).

The framework does not purport to say what reviews should be, nor does it capture all the existing knowledge on reviews. Rather, it seeks to specify the features that are agreed on by the major writers and which might be deemed essential to successful reviews. It also identifies areas of significant disagreement which could be expected to influence the choice of review type by a developer.

\subsection{The Components of the SDTR Framework}

One of the major contributions of the SDTR framework is to organise and structure the existing knowledge on reviews. Review characteristics can be categorised as follows: review aims and benefits which list the claimed aims and benefits of reviews; human elements which describe the human aspects of reviews; review process which describes the overall review process; review output which describes the physical outputs from a review process; and other matters which covers anything else. These components are not necessarily mutually exclusive, and do not impose constraints on assembling new review types.

\subsection{Key Features and Variations of SDTR}

In this section, we discuss for each SDTR component, the key features (in italics), followed by the possible variations reviews can take. Table 1 lists the key features and the major variations using the SDTR components.

\section{Review Aims and Benefits}

A review is used to improve the quality of software. It is agreed that the major aim of reviews is to improve the quality of software. The key quantitative benefit is that all reviews aim for error detection. 
Variations to the quantitative benefits are error correction, reduction in cost of testing, reduction in maintenance costs, improved predictability and productivity, and the establishment of standards [1$3,5,6,8]$. Next, a review has nonquantitative benefits. It is agreed that nonquantitative benefits exist, but there is no agreement on a key nonquantitative benefit. This need not be that the authors totally disagree; rather they place different emphases on what they perceive reviews should be. Variations to the nonquantitative benefits include providing reliable technical information for project control, reviewer education and communication, improving staff competence, providing management staff appreciation, creating better service to users, creating a more professional development environment, providing training to staff, and the ability to salvage incomplete work due to staff changes $[1-3,5,6,8]$.

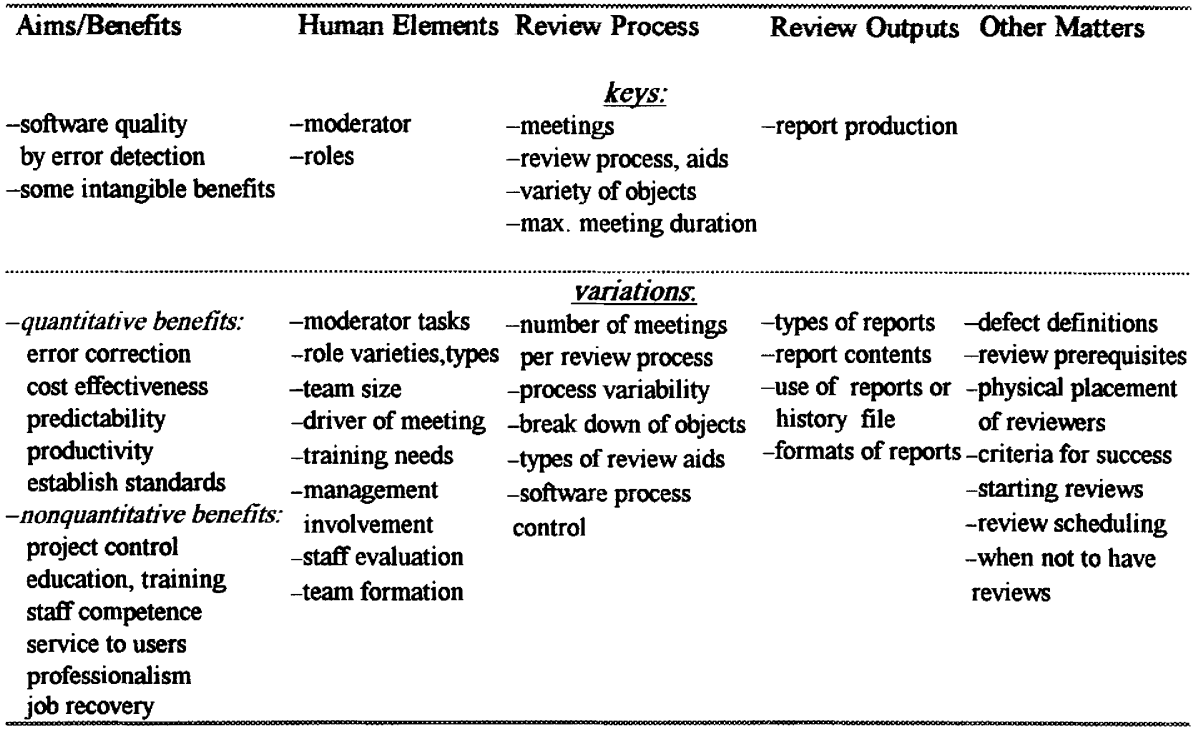

Table 1: The SDTR Framework - Key Features and Variations

Human Elements

A review must have a moderator or equivalent role. Each review type has a person responsible for the organisation and conduct of reviews (FI moderator, FWTR review leader, YSW coordinator). The variations lie in the moderator's tasks and task distribution. The FWTR review leader's tasks are similar to the FI moderator except that the recording is performed separately. The YSW recorder (secretary) produces the reports. Thus, the recording and report production are performed by either the moderator or recorder. Each reviewer must be assigned a role for the review meeting. Project and meeting roles are not differentiated. The authors agree that role assignment is essential in reviews. The variations lie in the variety of roles for each review type and the type of role represented (meeting or project role). Yourdon recommends three meeting roles. FWTR and FI have two meeting roles respectively.

One review variation is the review team size ( 4 for FI, at least 2 for FWTR, and 5 or 6 for YSW). The optimal team size is unknown. The driver of the FI meeting (absent in FWTR) is the author and the sequence of review discussions depends on this role. The equivalent YSW role is the presenter, whose task is to introduce the project. It is not clear to what extent the YSW presenter drives the meeting. Training requirements differ with review types. FI reviewers' training needs vary with roles; 
FWTR reviewers train by experience; and the training issue is not mentioned in YSW. There is some agreement that line supervisors should not be present at reviews. However, management can gain useful information depending on the distribution of reports. Yourdon suggests using reviews for staff evaluation. Fagan advises against it. The attitude of FI management and their acceptance of FI are important for success. Fagan also indicates when management is permitted to attend review meetings. Freedman and Weinburg, and Yourdon discuss the importance of using the same team for a project, over a period of time, for building effective teams.

\section{Review Process}

A review process must contain at least one meeting. A meeting is a formal physical gathering of people to carry out some common tasks. It requires some planning and organisation to ensure smooth running. The FI process contains up to two meetings: overview and review meetings (sometimes the overview is optional). The FWTR and YSW processes have one meeting only. A review process consists of a meeting, and pre-and post-review activities. The basic structure of all reviews consists of a meeting whereby reviewers agree on the set of errors present in the review object. The review meeting and the pre- and post-review activities make up the review process. The activities can change with the review object. A review process has aids to assist reviewers. For FI, error type checklists and frequently occurring errors are used. FWTR reviewer aids are more to help reviewers be good reviewers. YSW reviewer aids include standards, and recommendations for each role's responsibilities. A review process is applicable to various software development objects. However the objects suggested by the authors are different. The two types of YSW objects are operational prototypes, and product-related objects. FI objects are all product-related, whereas YSW and FWTR include operational prototypes. Variations include ways to break up an object. A review meeting usually lasts a maximum of two hours. It is agreed that a meeting should last 2 hours maximum. Only Yourdon recommends a minimum of half an hour.

A significant variation on the review process is in the use of reports to analyse review performance. Fagan recommends the use of statistical analysis on review data to improve future review processes. Freedman and Weinburg have similar ideas on review feedback and present practical advice on how the history file can be used [5]. Yourdon does not present any of these ideas.

\section{Review Output}

A review has as its output two or more reports. The report is central to the output of any review. It provides information that can be used to control software development. The variations in the reports lie in their contents, formats, and use. The review summary reports are usually of a fixed format and for management use. There is little consensus on report contents and why they are needed.

\section{Other Matters}

There is no obvious key feature in this category. However the variations are many. Defect definitions differ with review types. A defect in FI is an instance where the requirements and the exit criteria are violated. The standards against which errors in FWTR and YSW are considered are unknown. Yourdon implies that some defects are subjective (design) and are influenced by underlying assumptions. The prerequisites for each review type differ. FI requires well-defined development stages and entry/exit criteria to control the start/end of inspections. No prerequisites exist for FWTR, but YSW requires the use of structured development methodologies. Reviewers' placement is only discussed by Freedman and Weinburg (the recorder should be visible to everyone). FI's scheduling estimates are for systems programming only. No estimates are given for FWTR; Yourdon discusses some scheduling factors. Recommendations for starting reviews are varied. FI checklists can be developed from initial inspections. Freedman and Weinburg also give some tips for starting reviews [3]. Yourdon does not discuss it. Lastly, Yourdon discusses cases when it is not worthwhile having reviews. 


\section{CONCLUSIONS}

The SDTR framework identifies key features and differences among the seminal review types. The key features form the core of a normative theory of reviews. However, this theory (including the variations) is not as yet well tested. The theoretical status of the variations is less clear. Since they are not common to all review types, any given variation will be regarded as unnecessary by the proponents of the other review types. The categorisation of review attributes provides a foundation from which research can be conducted.

Systematic research of this kind is warranted for three reasons. First, there is limited knowledge of the actual performance of the three review types, and no substantial basis for comparison. Second, results from the study of group decision-making, highlight elements of reviews which are worthy of attention $[19,20]$. Third, Votta et al $[17,18]$ have begun to explore alternative approaches to inspections with encouraging results. The SDTR framework provides the basis for a sound understanding of the value of the elements of the core normative theory and its variants.

\section{REFERENCES}

1. Fagan M. E., 'Advances in software inspections', IEEE Transactions on Software Engineering, Vol SE12(7), 1986.

2. Fagan M. E., 'Design and code inspections to reduce errors in program development', IBM Systems Journal, Vol 15(3), 1979.

3. Freedman D. P., Weinburg G. M., Handbook of walkthroughs, inspections and technical reviews: evaluating programs, projects, and products, 3rd (Ed), Little, Brown and Company, 1982.

4. Pflegeer S. L., Software engineering: the production of quality software, 2nd (Ed), Macmillan, 1991.

5. Kim L. P. W., Sauer C., Jeffery R., 'A critical survey of software development technical reviews a nonmethod specific approach to sofware quality assurance', ITRC Report \#94/34, School of Information Systems, University of New South Wales, Apr 1994.

6. Weinburg G. M., Freedman D. P., 'Reviews, walkthroughs, and inspections', IEEE Transactions on Software Engineering, Vol SE-10(1), Jan 1984.

7. Yourdon E., 'Quality: what it means and how to achieve it', MIS, Feb 1993.

8. Yourdon E., Structured Walkthroughs, 4th (Ed), Yourdon Press, 1989.

9. Boehm et al B., Characteristics of software quality, New York, American Elsevier, 1978.

10. Myers G. J., 'A controlled experiment in program testing and code walkthroughs/inspections', Communications of ACM, Sep 1978.

11. Parnas D. L., Weiss D. M., 'Active design reviews: principles and practices', Journal of Systems and Software, Vol 7, pp 259-265, 1987.

12. Reeve J. T., 'Applying the Fagan inspection technique', Quality Forum, Vol 17(1), Mar 1991

13. Russell G. W., 'Experience with inspection in ultra large-scale developments', IEEE Software, pp25-31, Jan 1991.

14. Bush M., 'Improving software quality: the use of formal inspections at the Jet Propulsion Laboratory', IEEE, pp196-198, 1990.

15. Doolan E. P., 'Experience with Fagan's inspection method', Software - Practice and Experience, Vol 22(2), pp173-182, Feb 1992.

16. Weller E. F., 'Lessons from three years of inspection data', IEEE Software, pp38-45, Sep 1993.

17. Votta L. G. Jr., 'Does every inspection need a meeting ?', SIGSOFT'93, Dec 1993.

18. Porter L. G., Votta L. G., 'An experiment to assess different defect detection methods for software requirements inspections', forthcoming.

19. Levine R. E., Moreland R. L., 'Progress in small group research', Annual Review of Psychology, Vol(1), pp585-634, 1990.

20. ME Shaw, Group dynamics: the psychology of small group behaviour, McGraw-Hill, 1981. 